\title{
A suggestion to the article "Acute cerebellar edema after traumatic brain injury in a child. a case report"
}

\author{
Wang Bin ${ }^{1} \cdot$ Yang Tianmin ${ }^{1}$ \\ Received: 6 February 2020 / Accepted: 27 February 2020 / Published online: 12 March 2020 \\ (C) Springer-Verlag GmbH Germany, part of Springer Nature 2020
}

\section{Dear Editor:}

We have read with great interest the article "Acute cerebellar edema after traumatic brain injury in a child. a case report" written by Dr. Nader Hejrati and his colleagues [1]. This article has been translated into Chinese and published on the website "Neurosurgery News," which has attracted the attention of many neurosurgeons in China and prompted plenty of discussion.

In this article, an unusual case of acute cerebellar swelling after TBI with supratentorial contusions in a 10-year-old boy is described. In addition, the authors discussed the possible pathophysiological mechanism of this phenomenon. Some factors, such as posttraumatic sinus venous thrombosis, hypoosmolar hyponatremia, and disturbance of venous drainage, are proved unlikely in the case. Fortunately, with suboccipital decompressive and external ventricular drain surgery, bifrontal craniectomy, and ventriculoperitoneal shunt, the boy recovered with no evident neurological findings.

Nevertheless, we think that the immediate deterioration of the boy was due to the central hernia and tonsillar hernia caused by bifrontal contusion and edema. Supratentorial brain contusion with brain edema led to the increase in intracranial pressure, the downward displacement of the cerebral hemispheres, and the compression of venous sinuses at the base of the skull, which resulted in the disturbance of venous drainage. Diffusion-weighted imaging showed late signs of cerebral herniation, in terms of infarctions in the left anterior, medial, and posterior circulation, which also indirectly proved our view. In our hospital, there have been several similar cases of traumatic bifrontal contusions which were initially not serious but developed rapidly and often became abruptly severe and endangered the life of patients. We think it is better to perform bifrontal

Wang Bin

Lancet1979219@sina.com

1 Department of Neurosurgery, BenQ Medical Center, The Affiliated BenQ Hospital of Nanjing Medical University, Hexi Street 71, Nanjing 210019, China craniectomy firstly in this case, and suboccipital decompressive surgery can aggravate the downward movement of the brain.

The concept of central brain herniation originated in the $1980 \mathrm{~s}$ in various clinical reports. Central brain herniation demonstrates a top-down developmental process which first involves the diencephalon and then develops toward the mesencephalon, pons, and medulla oblongata [2]. These movements cause the brainstem to become compressed, pulled, and ischemic, which leads to swelling and hemorrhage. Early recognition and treatment of central brain herniation, due to its particular pathologic process, are vital for patient management. Effacement of the perimesencephalic cisterns is the most useful finding. Early symmetric miosis or slight disturbances of consciousness accompanied by irritability represent a key observation regarding the decision of surgery [3]. We recommend managing these patients with intensive care unit admission and early intracranial pressure monitoring. If they do deteriorate despite these measures, rapid bifrontal decompression can lead to good functional outcomes. The prognosis of this boy is good, which proves the authors' words: all the therapeutic options should be exhausted.

\section{Compliance with ethical standards}

Conflict of interest The authors declare no conflict of interest.

\section{References}

1. Hejrati N, Guzman R, Soleman J(2019) Acute cerebellar edema after traumatic brain injury in a child. a case report. Childs Nerv Syst. https://doi.org/10.1007/s00381-019-04418-3

2. Riveros Gilardi B, Muñoz López JI, Hernández Villegas AC, Garay Mora JA, Rico Rodríguez OC, Chávez Appendini R (2019) Types of cerebral herniation and their imaging features. Radiographics. 39(6): 1598-1610. https://doi.org/10.1148/rg.2019190018

3. Wu H, Yang SF, Qiu YM, Dai J, Li SQ, Zhang XH, Miao YF (2014) The diagnosis and surgical treatment of central brain herniations caused by traumatic bifrontal contusions. J Craniofac Surg 25(6): 2105-2108. https://doi.org/10.1097/SCS.0000000000001050

Publisher's note Springer Nature remains neutral with regard to jurisdictional claims in published maps and institutional affiliations. 